\title{
Transmission désordonnée et speckle
}

Faire une image, un hologramme, une mesure en métrologie, utiliser une pince optique pour manipuler une cellule, une macromolécule, propager un faisceau laser, conjuguer plusieurs faisceaux pour accumuler leur énergie en un point précis de l'espace à l'instant souhaité, oblige à propager ces faisceaux dans des milieux variés soumis à diverses contraintes. Ces faisceaux peuvent traverser non seulement des milieux gazeux, liquides ou solides dans lesquels des fluctuations de concentrations figées ou non sont susceptibles d'exister, mais aussi des interfaces immanquablement soumises à des rugosités d'échelles variées. Bref, la lumière rencontre sur son chemin de nombreux obstacles avant d'arriver sur le capteur, la photodiode à avalanche, une simple caméra, un spectroscope ou un imageur multispectral.

Nous nous intéresserons dans cet article à décrire la principale conséquence d'une interaction avec un milieu désordonné, à savoir l'apparition d'un bruit ou d'un signal nommé « speckle », celui-ci pouvant être objectif ou subjectif.

\section{Jean TABOURY}

Laboratoire Charles Fabry

Institut d'Optique Graduate School

jean.taboury@institutoptique.fr

\section{Le speckle objectif}

Lorsque l'on observe sur un écran la figure de speckle générée par la diffusion d'une onde sur un objet diffusant à une distance suffisante, on peut remarquer que la structure moyenne du grain de speckle - encore appelé tavelure ou image d'aspect vermiculé - est très dépendante de la forme même de l'objet. C'est ce que l'on appelle une image de speckle objectif. Dans cette partie nous allons préciser quelques propriétés spatiales du champ et de l'éclairement présents dans le voisinage immédiat d'un point de l'espace noté $M$.

Nous mettrons ainsi en évidence l'effet de l'étendue spatiale des sources secondaires sur la forme en 3 dimensions du grain du speckle objectif. Pour cette étude, nous évaluerons l'expression de la corrélation du champ en $M$ avec le champ présent dans son voisinage en $M^{\prime}$. L'expression de cette corrélation prendra une forme simple lorsque I'angle solide sous lequel I'objet diffusant est vu du point $M$ sera relativement petit. Dans le cas d'une source diffusante à symétrie circulaire, nous en déduirons une estimation des dimensions transversales et longitudinales du grain de speckle. Ces caractéristiques sont alors similaires à celles que I'on peut rencontrer dans l'image d'un objet diffusant quelconque obtenue par un système optique à pupille circulaire lorsque l'objet est soumis à un éclairage spatialement cohérent. L'image est alors entachée d'un bruit dû à un autre type de speckle, appelé speckle subjectif. Le grain de ce speckle subjectif possède des caractéristiques en dimensions qui ne dépendent plus de l'objet mais uniquement de la forme de la pupille.

Ce speckle subjectif se rencontre également dans d'autres modalités d'imagerie, comme l'imagerie ultrasonore médicale et l'imagerie radar ou sonar à synthèse d'ouverture.

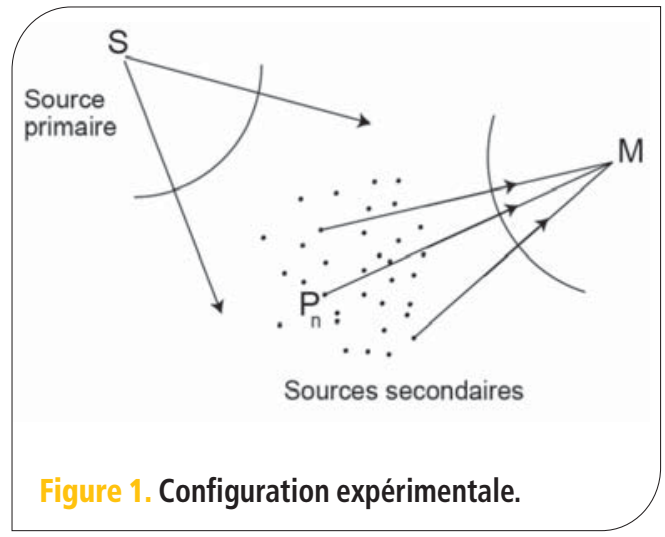

\section{Configuration expérimentale}

Considérons une distribution de sources secondaires discrètes (figure 1).

Chaque source secondaire est excitée par une source primaire $S$ ponctuelle et monochromatique de vecteur d'onde $k=\frac{2 \pi}{\lambda}$ (par exemple un laser épuré).

Ces sources secondaires émettent par conséquent des ondes synchrones dans l'espace.

\section{Effet de l'étendue de la source diffusante sur les propriétés spatiales du champ et de l'éclairement}

Le champ au point $M$ sera le résultat de l'interférence de $\mathrm{N}$ ondes notées $U_{\mathrm{n}}$ provenant des $\mathrm{N}$ sources secondaires éclairées par la source primaire $S^{1}$.

$$
U(M)=\sum_{n}\left(U_{s} \frac{\exp \left(i k S P_{n}\right)}{S P_{n}} A_{n} \frac{\exp \left(i k P_{n} M\right)}{P_{n} M}\right)
$$

Dans cette expression, $S P_{n}$ et $P_{n} M$ représentent les distances séparant les points

correspondants.
Appelons $B n$ la quantité $U_{s} \frac{\exp \left(i k S P_{n}\right)}{S P} A_{n}$ où $A_{n}$ traduira l'effet du centre diffuseur produit sur l'amplitude complexe de l'onde émise et un éventuel déphasage variable suivant l'état de polarisation de l'onde incidente.

\section{Que peut-on attendre du champ} et de l'éclairement au point $M$ ?

La distribution aléatoire des centres diffuseurs dans le volume d'un objet ou dans l'épaisseur de son revêtement 


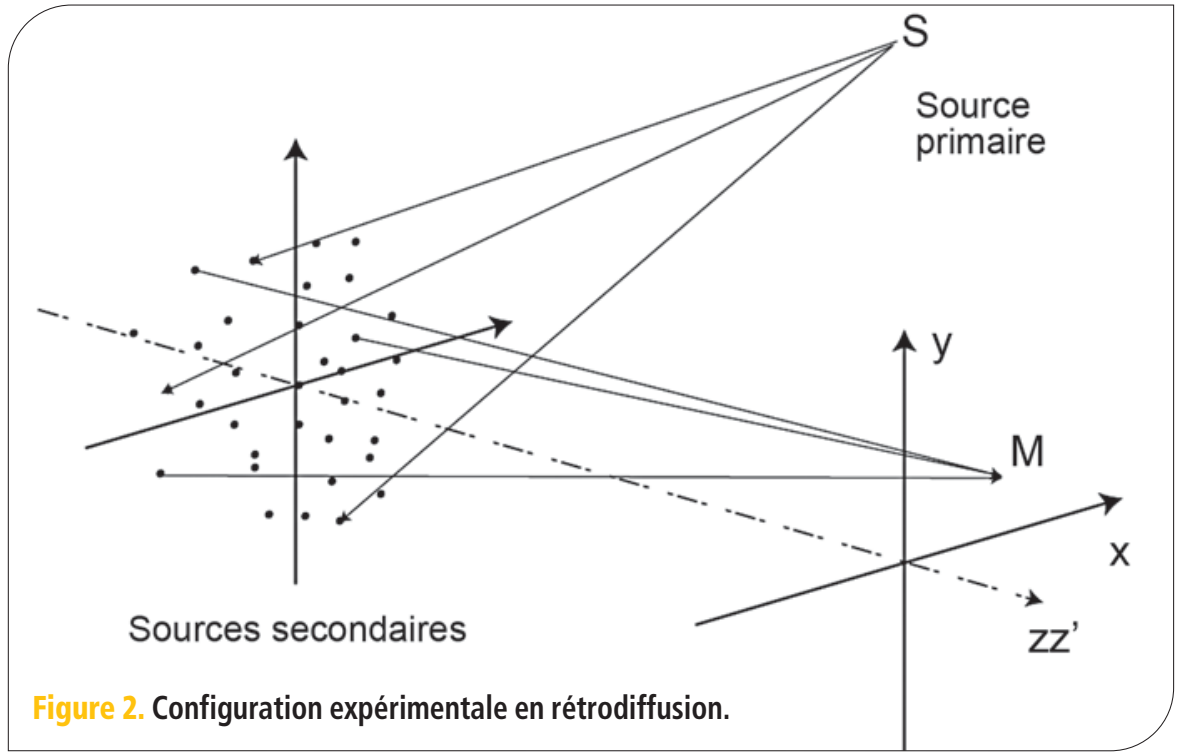

donne à cette variable complexe des propriétés statistiques particulièrement intéressantes. L'une de ces propriétés caractérise la distribution des différences de marche entre tous les rayons qui viennent interférer en $M$. À l'échelle de la longueur d'onde, il est facile de se convaincre que cette distribution modulo $2 \pi$ sera uniforme sur l'intervalle $[-\pi,+\pi]$.

En conséquence, la valeur moyenne attendue du champ complexe en ce point $M$ de l'espace est nulle. II n'en est pas de même de son module, qui suit une loi de Rayleigh alors que sa phase est uniformément repartie sur le cercle trigonométrique. On dira dans ce cas que le speckle est pleinement développé.

La deuxième conséquence réside dans la densité de probabilité attendue de l'éclairement mesurable en $M$. Cette densité de probabilité suit une exponentielle décroissante à condition que la mesure soit faite sur une surface très petite à l'échelle du grain de speckle et dans un temps suffisamment court si des mouvements relatifs des sources secondaires existent. II s'en suit que le rapport de l'écart type à la moyenne est égal à 1.

\section{Évaluation de la corrélation des champs dans le voisinage du point $M$}

Pour une réalisation donnée, si le champ et l'éclairement suivent les lois décrites précédemment, il est intéressant de prévoir le comportement de ces grandeurs lorsque l'on se déplace légèrement autour du point $M$ à un instant précis. Pour répondre à cette question, il sera nécessaire d'évaluer la corrélation du champ présent en $M$ avec celui présent dans son voisinage $M^{\prime}$ (figure 2).

Cela revient à estimer le moment composé d'ordre $2\left\langle U(M) U^{*}\left(M^{\prime}\right)\right\rangle$ où l'étoile représente le complexe conjugué. En remplaçant $U(M)$ et $U^{*}\left(M^{\prime}\right.$ ) en fonction de $B_{n}$ nous obtenons une expression que I'on peut développer au premier ordre dans une hypothèse satisfaisant l'ap-

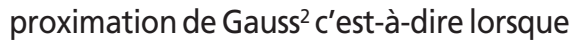
I'on considère le point $M$ situé à une distance $d$ suffisamment éloignée de I'objet. Cette approximation permet de caractériser simplement l'aspect spatial de cette figure de speckle.

\section{Corrélation transverse}

Dans un souci de simplification, les points $M$ et $M$ ' sont situés dans un même plan à la distance $d$ des sources que nous considérerons elles-mêmes comme distribuées dans un plan parallèle au précédent. L'équation se simplifie et nous admettrons qu'elle peut prendre la forme suivante:

$\left\langle U(M) U^{*}\left(M^{\prime}\right)\right\rangle=\sum_{n}\left(B_{n} B_{n}^{*} \exp \left(-i \frac{k}{d}\left(x_{p_{n}}\left(x_{M^{\prime}}-x_{M}\right)+y_{p_{1}}\left(y_{M^{\prime}}-y_{M}\right)\right)\right)\right)$

Là encore, afin de clarifier la démonstration, nous considérons que la luminance apparente $L(x, y)$ de cette distribution de sources dans le voisinage d'un point $P$ peut être évaluée à partir de la densité locale de ces sources.
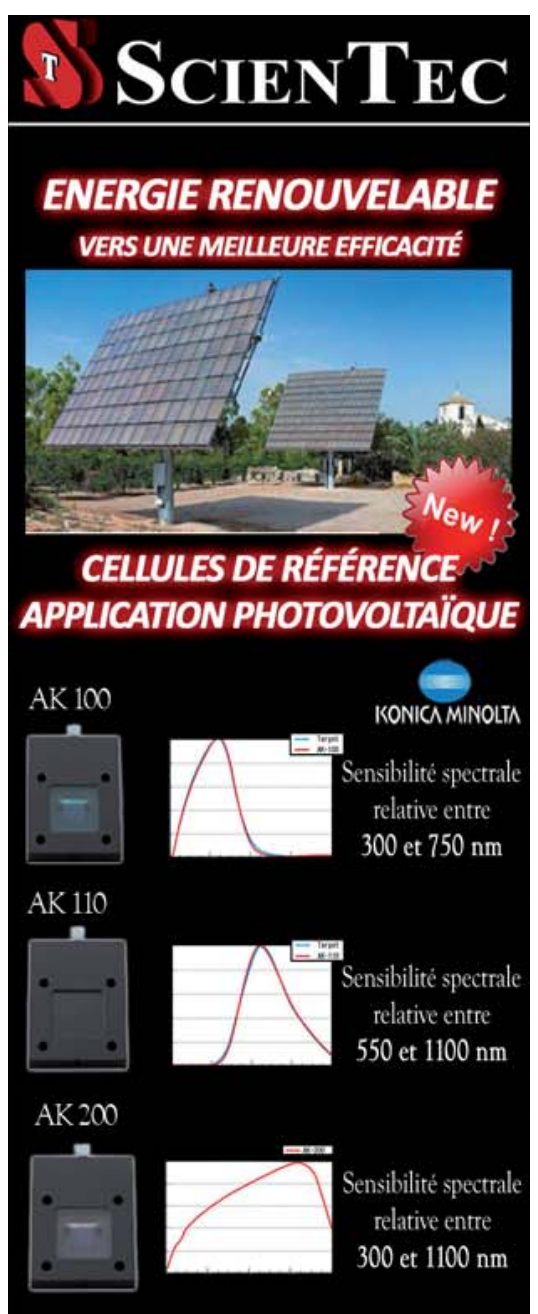

Régler avec précision l'éclairement lumineux des simulateurs solaires

Essentiel pour évaluer les caractéristiques des cellules photovoltaĩques et respecter les normes de mesures internationales

- Conforme aux normes JIS

- Perte spectrale inférieure à $1 \%$

- Amélioration de la durabilité

\section{ScienTec,} vous propose en complément

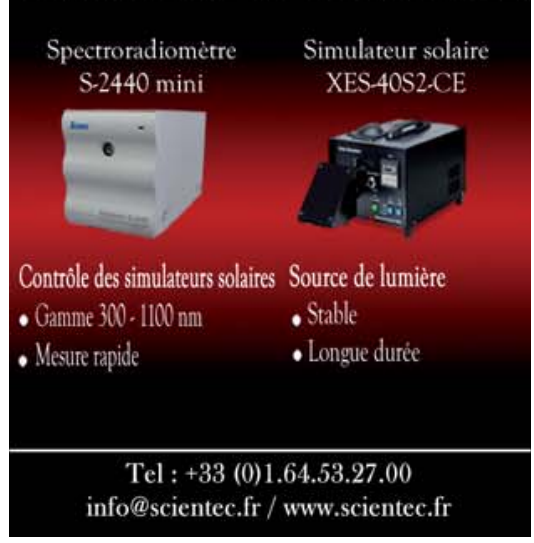



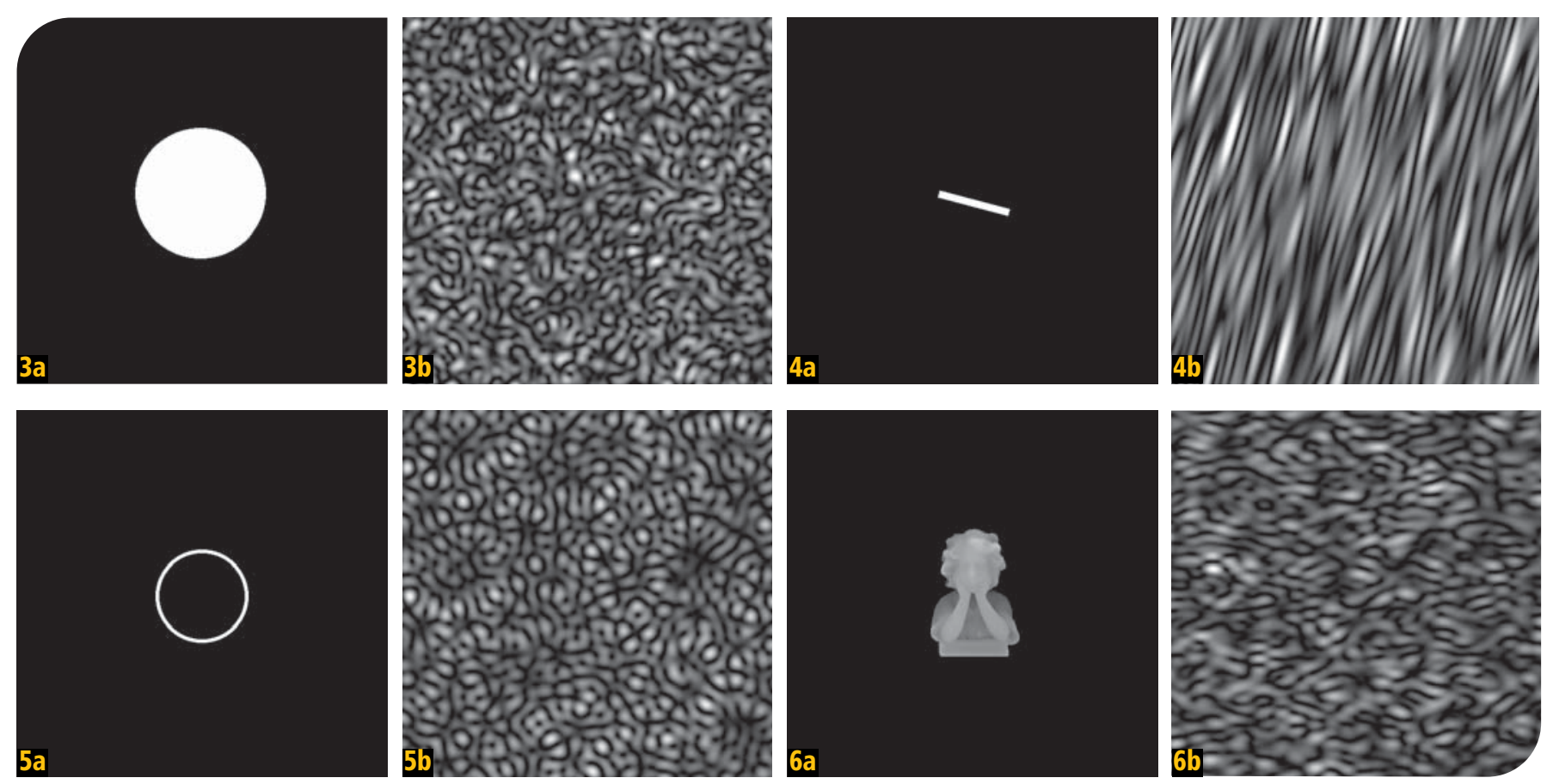

Cette somme discrète peut alors s'étendre à une intégrale continue dans le plan contenant les sources secondaires. Dans ces conditions, elle conduit à un résultat remarquable d'une très grande importance en optique : la corrélation du champ dans le voisinage du point $M$ s'identifie à la transformée de Fourier de la luminance apparente de la scène diffusante:

$$
\left\langle U(M) U^{*}\left(M^{\prime}\right)\right\rangle \propto T F[L(x, y)]_{\frac{\left(x^{\prime}-x\right)}{\lambda d} \cdot \frac{\left(y^{\prime}-y\right)}{\lambda d}}
$$

Le degré de cohérence du champ s'obtiendrait par simple normalisation de cette équation.

\section{Exemple}

Afin de se fixer les idées, considérons une pastille circulaire de diamètre $\Phi$ sur laquelle une distribution uniforme de granules de très petites dimensions constituera l'ensemble des sources secondaires. Cette pastille est éclairée par un laser de longueur d'onde $\lambda$ et le champ est observé à la distance $d$. L'application du résultat précédent nous permet d'évaluer le diamètre caractéristique de la corrélation transverse. On montre que ce diamètre est de l'ordre de grandeur de $\lambda \frac{d}{\Phi}$. Un calcul différent nous amènerait à évaluer la corrélation axiale du champ lorsque l'on s'éloigne de l'objet. Son évaluation conduit à une longueur de l'ordre de grandeur de $\lambda\left(\frac{d}{\Phi}\right)^{2}$. Le volume dans lequel le champ possède une corrélation certaine est dans ce cas un ellipsoïde allongé. La connaissance de ces caractéristiques spatiales est importante pour des applications dans lesquelles ce champ doit interférer avec une onde de référence comme en holographie classique ou numérique. Dans ce cas, cet aspect désordonné de la répartition du champ et de l'éclairement doit être considéré comme un véritable signal car il renferme une information sur l'objet et ne peut être confondu avec un bruit.

On remarque que l'on retrouve également ce concept de volume de corrélation ou de cohérence dans l'analyse du fameux théorème de Zernike et van Cittert.

\section{Moment d'ordre 2 de l'éclairement}

Étudier le moment d'ordre 2 de l'éclairement ou sa corrélation $\left\langle I(M) I\left(M^{\prime}\right)\right\rangle$ où l'éclairement $I(M)$ s'identifie au produit du champ par son complexe conjugué, permettra d'estimer la taille caractéristique des grains de speckle observables sur l'écran dont nous avons parlé antérieurement. La simple application d'un théorème sur les moments gaussiens centrés d'ordre 4 permet d'évaluer rapidement la corrélation entre les éclairements de deux points voisins, I'un en $M$ l'autre
Figure $3(a, b)$. Speckle objectif produit par un objet diffusant uniforme limité par un diaphragme circulaire.

Figure $4(\mathrm{a}, \mathrm{b})$. Speckle objectif produit par un objet diffusant uniforme limité par un diaphragme rectangulaire dont la longueur est dix fois sa largeur.

Figure $5(a, b)$. Speckle objectif produit à grande distance par un objet diffusant uniforme formé d'un anneau circulaire.

Figure $6(a, b)$. Speckle objectif produit par une statue située à grande distance.

en $M^{\prime}$. Ce résultat que nous ne démontrerons pas ici, fait intervenir la corrélation entre les champs aux points $M$ et $M$ ' que nous avons déjà présentée :

$\left(\left\langle I(M) I\left(M^{\prime}\right)\right\rangle-I(M) I\left(M^{\prime}\right)\right) \propto\left|T F[L(x, y)]_{\frac{\left(x^{\prime}-x\right)}{\lambda d}, \frac{\left(y^{\prime}-y\right)}{\lambda d}}\right|^{2}$

Les fluctuations spatiales de l'intensité, caractéristiques de ces images de speckle vermiculées, ont des dimensions tout à fait comparables avec celles que nous avons évoquées précédemment.

Une des applications les plus simples et élémentaires de ce résultat repose sur l'observation au travers d'un dépoli du speckle provoqué par l'impact d'un faisceau laser focalisé sur une cible (figures 3 à 6). Lorsque la focalisation sera bonne, le grain observé sur le dépoli sera le plus gros. Si de l'astigmatisme entache le 
point focal, l'orientation du grain renseignera tout de suite sur l'aberration rencontrée.

\section{Effet du mouvement désordonné des sources secondaires sur l'évolution temporelle du speckle}

Considérons un ensemble de sources secondaires soumises à une simple agitation thermique. Citons comme exemple le cas du mouvement brownien visible au microscope, comme les grains de pollen en suspension dans l'eau. Pour des structures diffusantes de taille beaucoup plus petite comme les macromolécules biologiques, les fluctuations temporelles du speckle seront beaucoup plus rapides et riches en informations sur des paramètres physiques comme les constantes de diffusion de ces molécules, en fonction par exemple de leur environnement aqueux ou du pH. Pour d'autres configurations, comme l'étude du séchage de peintures ou l'évaluation de la viscosité de certains cosmétiques, ou le suivi de cinétiques physico-chimiques, l'analyse du temps de décorrélation du speckle renseignera sur les paramètres physico-chimiques mis en jeu. Lorsque les échelles de description des fluctuations de concentration ou d'indice seront à beaucoup plus grande échelle, d'une fraction de centimètre à quelques mètres, on ne parlera plus de diffusion mais d'aberrations dynamiques du front d'onde et on utilisera des analyseurs de front d'onde (voir l'article "L'analyse de surface d'onde " de Jérôme Primot, publié dans le numéro 52 de Photoniques) et des optiques adaptatives pour traiter ce genre de problème. Dans la suite, nous nous restreindrons à la diffusion simple afin de décrire le principe de base de l'effet de cette dynamique sur la figure de speckle sans aborder le domaine des grandes échelles.

Considérons une structure désordonnée dans laquelle les centres diffuseurs sont animés d'une distribution de vitesse gérée par une loi de type Boltzmann. Appelons $\vec{V}_{n}$ la vitesse au point $P_{n}$. Durant le temps $\tau$, la distance parcourue sera $P_{n} P_{n}^{\prime}=V_{n} \tau$. Durant cet intervalle de temps, le champ au point $M$ varie d'une petite quantité que l'on peut exprimer par un développement limité au premier ordre de l'équation exprimant le champ au point $M$ :

$U^{\prime}(M)=\sum_{n} U_{n}(M)\left(1+i k \overrightarrow{P_{n} P_{n}^{\prime}} \cdot \overrightarrow{\operatorname{grad}}\left(S P_{n}+P_{n} M\right)\right)$

Dans cette expression nous pouvons remarquer que le terme additionnel revient à déphaser le champ $U_{n}(M)$ émis en chaque point à l'instant $t$ d'une petite quantité $\phi_{n}$ proportionnelle au produit scalaire du gradient et du vecteur déplacement du point $P_{n}$ :

$$
U\left(M^{\prime}\right) \approx \sum_{n} U_{n}(M) \exp \left(i \phi_{n}\right)
$$

On peut montrer que ce déphasage $\phi_{n}$ peut encore s'écrire sous la forme suivante :

$$
2 \cos \left(\frac{\alpha}{2}\right) V_{n} \cos (\beta) \tau
$$

Ce déphasage fait intervenir l'angle $\alpha$ entre le rayon incident et le rayon diffusé au point $P_{n}$ comme indiqué dans la
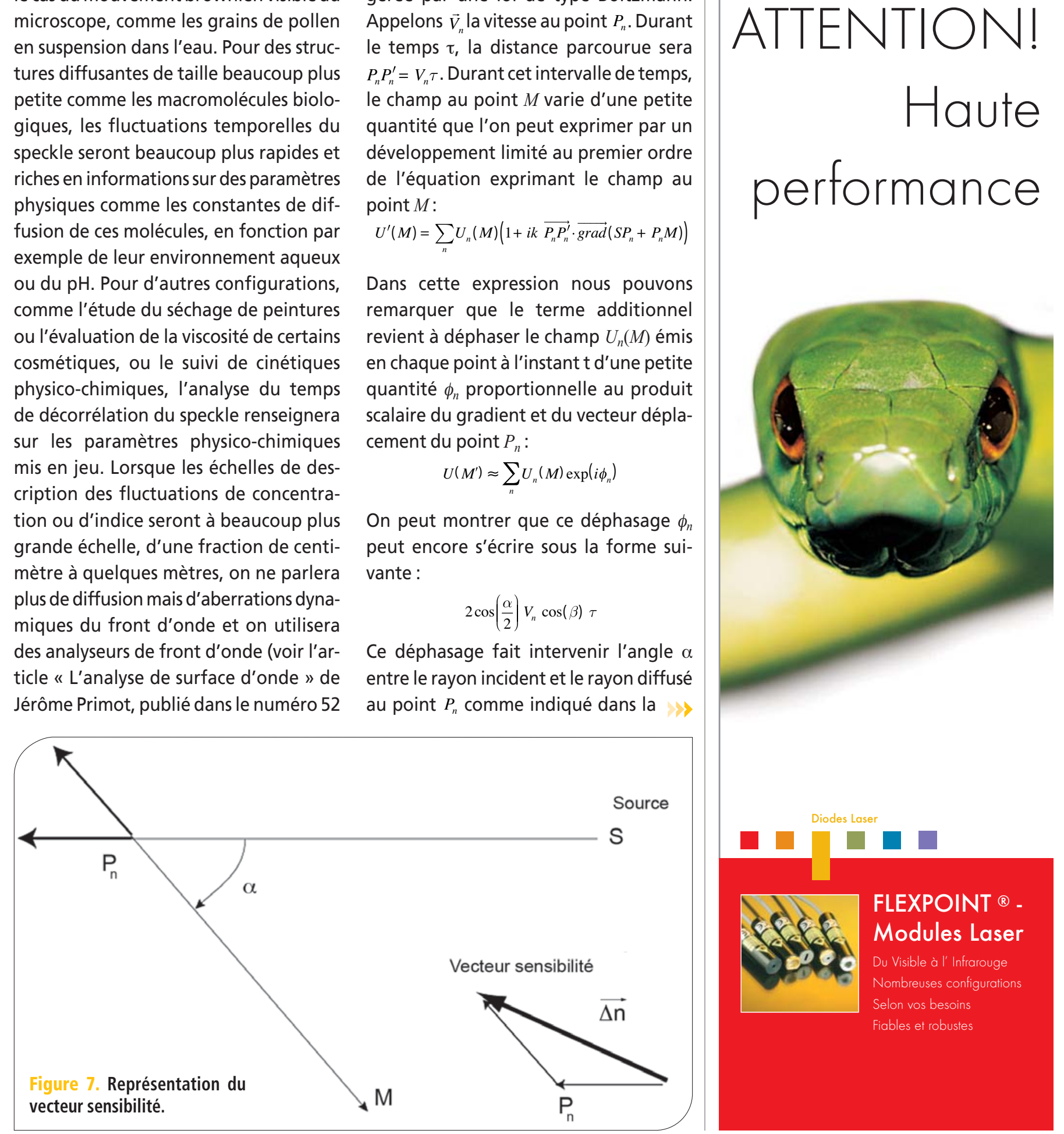
figure 7. Le vecteur $\overrightarrow{\Delta n}$ représente le gradient de la somme. Ce vecteur est encore appelé vecteur sensibilité. L'angle $\beta$ mesure l'angle entre le vecteur $\overrightarrow{\Delta n}$ et le vecteur $\vec{V}_{n}$.

La quantité $\phi_{n}$ dépend non seulement de la vitesse de déplacement du point $P_{n}$, de l'intervalle de temps $\tau$, mais aussi de la longueur du vecteur $\overrightarrow{\Delta n}$ et de l'angle $\beta$ entre le vecteur vitesse et le vecteur sensibilité.

À une distance suffisante de l'objet, la norme du vecteur $\overrightarrow{\Delta n}$ peut être considérée comme pratiquement indépendante de la position du point $P_{n}$. L'expression de la phase $\phi_{n}$ montre ainsi clairement que la sensibilité au déplacement sera très différente suivant la direction d'observation. C'est cette propriété qui confère à cette technique son très large domaine d'application. Dans une configuration de rétrodiffusion, la sensibilité au déplacement sera maximale et permettra d'ausculter des échelles de fluctuation de l'ordre de grandeur de la demi-longueur $d$ 'onde à des échelles de temps très court de la microseconde à la nanoseconde. En revanche, dans I'axe des rayons incidents, la décorrélation de la phase entre les différentes parties du milieu testé décuplera de plusieurs ordres de grandeur les distances et les durées de corrélation. La propagation dans l'air surchauffé des jours de canicule donne une idée du temps de corrélation des figures de speckle qui se rapproche alors plutôt de l'ombroscopie et qui est connu sous la terminologie d'« ombre volante ».

\section{Le speckle subjectif}

Pour terminer, nous ferons simplement allusion à l'imagerie cohérente. Lorsque la source primaire est ponctuelle ou de faible étendue, le système d'imagerie est linéaire en amplitude. Il se forme en premier une image en amplitude de l'objet dont le capteur ne pourra enregistrer que le carré de son module. Cette superposition cohérente des réponses percussionnelles en amplitude, pondérées par les propriétés de l'objet, forme un réseau désordonné de franges d'interférences dont les positions relatives des maximums

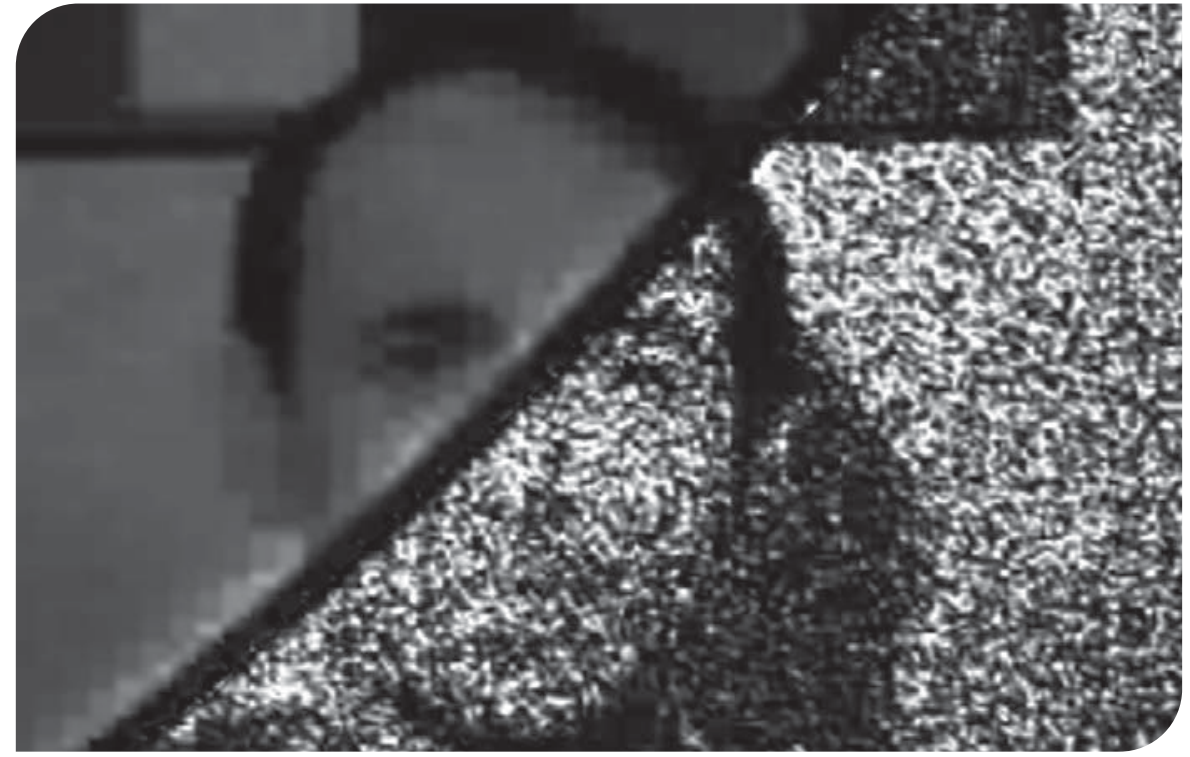

Figure 8. Image d'une diapositive éclairée par un laser. La partie gauche, plus nette, a été vue au travers d'un dépoli tournant à grande vitesse pour que de nombreuses images de speckle subjectif décorrélées se superposent sur le capteur durant le temps de pose. La taille du grain de la partie droite est directement liée à l'inverse du diamètre de la pupille du système d'imagerie.

et des minimums dépendront des différences de marche relatives entre les rayons. Ce bruit, dû à ces interférences dans cette image vermiculée, est appelé speckle subjectif.

On comprend ainsi qu'une modification des propriétés physiques de la surface aura de lourdes conséquences sur le résultat de cette figure d'interférence. II suffit pour cela de regarder la partie droite de l'image d'une simple diapositive éclairée par un faisceau cohérent pour s'en convaincre (figure 8). Quant à sa partie gauche, un disque dépoli tournant rapidement et situé en amont de la diapositive a permis durant le temps de pose d'une fraction de seconde de brouiller presque totalement l'effet de ces franges d'interférence désordonnées. Dans ces conditions, le bruit de speckle subjectif a totalement disparu. On montrerait que ce bruit, estimé par la racine de la variance de l'image dans une zone homogène, décroît comme la racine du nombre d'images de speckle décorrélées. Le système d'imagerie est alors devenu linéaire en éclairement dans sa partie gauche. On montrerait également que le support du contenu spectral de la partie droite de l'image et de son bruit n'est autre que le support de la FTM de I'optique. Un filtrage passe bas réduirait bien sûr ce bruit, mais au détriment du détail contenu dans l'image.

Les conditions d'éclairage sont déterminantes, et sa maîtrise la principale méthode à retenir pour réduire ce type de bruit. Pour que ce bruit disparaisse, il importe que l'objet soit spatialement cohérent. Cela est vrai uniquement lorsque l'ouverture numérique sous laquelle l'objet voit la source primaire est supérieure à l'ouverture sous laquelle l'objet voit la pupille du système d'imagerie. Si par un beau ciel bleu très pur vous regardez une pièce de monnaie ou la surface diffusante d'une plaque d'aluminium dépolie, vous ne serez pas sans remarquer que l'image de l'objet est entachée d'une texture vermiculée dont l'irisation colorée est caractéristique. Cette texture possède un aspect mouvant indépendant de l'objet. C'est la raison pour laquelle ce bruit est dit subjectif.

\section{Pour en savoir plus...}

B. Chu. Laser Light Scattering: Basic Principles and Practice. Academic Press, 2e édition, 1991.

J.-W. Goodman. Introduction à I'optique de Fourier et à l'holographie. Masson,Paris, 1972.

J.-W. Goodman. Speckle Phenomena in Optics. Roberts \& Company Publishers, Greenwood Village, C0, 2007, 406 pages. 